\title{
Empirical Orthogonal Representation of Time Series in the Frequency Domain. Part I: Theoretical Considerations ${ }^{1}$
}

\author{
JoHN M. WALlaCE \\ Dept. of Atmospheric Sciences, University of Washington, Seattle 98105 \\ AND Robert E. Dickinson \\ National Center for Atmospheric Research, ${ }^{2}$ Boulder, Colo. 80302 \\ (Manuscript received 11 February 1972, in revised form 19 May 1972)
}

\begin{abstract}
Difficulties in using conventional cross-spectrum analysis to explore atmospheric wave disturbances have indicated the need for some extension of the usual technique. It is suggested here that the eigenvectors of the cross-spectrum matrix be used for interpreting such data. The method is analogous to the use of empirical orthogonal functions applied to band-pass filtered time series. However, the eigenvectors of the crossspectrum matrix contain additional information concerning phase which is not available from the eigenvectors of the covariance matrix. It is possible to generate a new set of time series which are mutually uncorrelated within a pre-selected frequency interval and which have the same combined variance in the frequency interval as the original set of time series. These new series are obtained by applying the eigenvectors of the cross-spectrum matrix to a set of complex time series involving the original time series and their time derivatives. The application and physical interpretation of the technique are discussed. Examples of the technique applied to atmospheric data are given in a second part of this study.
\end{abstract}

\section{Introduction}

During the past few years, cross-spectrum analysis of time series data has seen extensive use as a technique for describing large-scale disturbances in the tropical atmosphere. The objective of these studies has been to infer the relationships between different atmospheric variables (e.g., wind and temperature) and various spatial relationships between the waves present within a particular frequency interval. A "wave" is defined observationally by the presence of statistically significant spatial and inter-parameter correlations which can be interpreted in terms of a diagnostic physical model.

The general procedure followed in these investigations has been to compute the coherences and phase relationships between various parameters in one or more frequency bands which had been selected for intensive investigation. The time series of one parameter (e.g., meridional wind component at some specified level) is designated as a "base series" and the coherences and phase relationships are computed between this series and those of other parameters. This is analogous to the technique employed in compositing studies (e.g., Reed and Recker, 1971) wherein compositing is done relative to one reference parameter.

\footnotetext{
${ }^{1}$ Contribution No. 256, Department of Atmospheric Sciences, University of Washington.

2 The National Center for Atmospheric Research is sponsored by the National Science Foundation.
}

When more than one type of wave structure is present in the same frequency band, there is considerable difficulty in interpreting the cross-spectrum data. There is no way of determining how many significant wave structures are present, and what is the relative contribution of each wave type to the variance spectra. Some of these difficulties in interpretation have been discussed by Wallace (1971).

Even when only a single wave is present, there are some technical objections to the use of a base series: 1) In computations of wave amplitudes, this method always produces a bias in favor of the base series. If the coherence between various parameters and the base series is not large, this can result in considerable distortion in the pattern of wave amplitude. 2) The method does not exploit the information contained in the cross spectra between parameters other than the base series. These latter difficulties are apparently not overly serious since various studies using different base series have produced similar results.

To summarize the kind of data available, we may have data at $L$ different levels for $M$ different stations and $N$ different atmospheric variables for a total of $n=L \times M \times N$ variables. These variables define an $n$-dimensional vector-valued time series which can be subjected to cross-spectrum analysis. For any given frequency band, the cross spectra between all possible pairs of variables can be displayed in an $n \times n$ matrix, 
the cross-spectrum matrix. The diagonal elements of this matrix are the power spectral estimates obtained by crossing each time series with itself. The off-diagonal elements are complex, the real part being identified with the co-spectrum and the imaginary part with the quadrature spectrum.

There is a need for some objective way to define the number of significant wave disturbances present in certain frequency intervals and to separate the total disturbance field into individual wave components. It is also desirable that this method should fully exploit the statistical information contained in the crossspectrum matrix.

The approach we have adopted to achieve these objectives is to express the vector time series as a linear. combination of the eigenvectors of the cross-spectrum matrix for the frequency interval of interest. This procedure is closely related to the application of the empirical orthogonal function analysis (Lorenz, 1956) to simultaneous multiple time series which have been band-pass filtered to eliminate all components outside the frequency band being considered. However, here we use the phase as well as the amplitude of the correlation between the series so the procedure is appropriately referred to as complex eigenvector analysis. Groves and Hannan (1968) have described a related procedure for decomposing a cross-spectrum matrix into components coherent with other time series and a residual matrix.

The present study consists of two parts. In Part I we present some of the mathematical properties of the technique and discuss the application of the method. Part II (Wallace, 1972) applies the method to detailed analysis of tropical_wave disturbances.

\section{Application of empirical orthogonal functions}

While applications generally involve sampling over discrete intervals for a finite length of time, we describe here the theory formally in terms of a random time series infinite in length. The time series has components $u_{j}(t)$. A covariance matrix $\mathbf{U}_{j l}$ can be generated by

$$
\dot{\mathbf{U}}_{j t}=\left\langle u_{j}(t) u_{l}(t)\right\rangle
$$

where the angular brackets denote an ensemble average which is in practice usually replaced by a time average.

In the method of empirical orthogonal functions we introduce new variables $z_{i}(t)$, which are statistically uncorrelated. This is done by finding the eigenvectors of the symmetric matrix $\mathbf{U}_{j l}$. The matrix element $e_{i j}$ formed from the $j$ th element of the $i$ th eigenvector gives the desired transformation

$$
z_{i}(t)=\sum_{j} e_{i j} u_{j}(t)
$$

We shall use $i$ and $k$ subscripts throughout to denote the $i$ th or $k$ th eigenvector while $j$ and $l$ are used to denote $j$ th or $l$ th elements of parameter space. The covariance matrix of the $z_{i}$ is diagonal, i.e., if we use the notation $\delta_{i k}=1$ if $i=k, \delta_{i k}=0$ if $i \neq k$, then

$$
Z_{i k}=\left\langle z_{i}(t) z_{k}(t)\right\rangle=\delta_{i k} \lambda_{i},
$$

where $\lambda_{i}$ are the eigenvalues of $\mathbf{U}_{j l}$, which are real because $\mathbf{U}_{j l}$ is symmetric.

For reasonable normalizations of the $u_{j}(t)$ as discussed in a later section, we can measure the relative importance of the $i$ th component of the new time series $z_{i}(t)$ by its contribution to the trace of the covariance matrix

$$
\mathrm{T}=\sum_{j} U_{j j}=\sum_{i} Z_{i i}=\sum_{i} \lambda_{i}
$$

which is simply the sum of the variances of the $u_{j}$. In other words, $z_{i}(t)$ contributes the fraction $\lambda_{i} / T$ of the total variance of the series. Often most of the variance in the time series is carried by only a few $z_{i}$ with relatively large $\lambda_{i}$. It is convenient to order the $z_{i}(t)$ in order of decreasing magnitude of the $\lambda_{i}$.

In the foregoing discussion we have indicated how the original time series $u_{j}(t)$ can be expressed in terms of a new time series of uncorrelated variables $z_{i}(t)$,

$$
u_{j}(t)=\sum_{i=1}^{n} e_{i j \sigma_{i}}(t)
$$

This is accomplished by means of a linear transformation of the original series making use of the eigenvectors of its covariance matrix. The eigenvector component $e_{i j}$ determines the sign and relative weight of the contribution which the $j$ th input variable makes to the $i$ th transformed variable. Thus, each eigenvector $\mathbf{e}_{i}$ determines a structural relationship between the input variables in a manner completely analogous to the sine and cosine functions of a Fourier series expansion in the parameter domain.

Properties of the empirical orthogonal representation are summarized as follows:

1) The eigenvectors are orthogonal in the parameter domain because the covariance matrix is symmetric. They can be normalized to unity so that

$$
\sum_{j} e_{i j} e_{k j}=\delta_{i k}
$$

2) The elements of the transformed time series are uncorrelated at zero lag [cf. Eq. (3)].

3) The variance explained by the $i$ th empirical orthogonal function is given by

$$
\left\langle z_{i}{ }^{2}\right\rangle=\lambda_{i},
$$

where $\lambda_{i}$ is the $i$ th eigenvalue of the covariance matrix $\mathbf{U}_{j l}$. Summing over all the eigenvalues, we get the trace of $Z_{i k}$ which is the total variance of all the variables.

4) The fraction of the variance of the $j$ th parameter explained by the $i$ th empirical orthogonal function is given by the square of the coefficient of the 
correlation between the $u_{j}$ and $z_{i}$ series, which can be expressed in the form

$$
\gamma_{i j}=\frac{\left\langle u_{j z_{i}}\right\rangle^{2}}{\left\langle u_{j}{ }^{2}\right\rangle\left\langle z_{i}{ }^{2}\right\rangle}=\frac{e_{i j}{ }^{2} \lambda_{i}}{\left\langle u_{j}{ }^{2}\right\rangle} .
$$

From this relation one can compute the amplitude of the fluctuations of each parameter $u_{j}$ in each of the empirical orthogonal functions.

\section{Frequency domain}

We indicate here how the theory just summarized is modified by using the eigenvectors of the cross-spectrum matrix in place of the covariance matrix to represent the parameter space structure of a multiple time series. The eigenvectors of the cross-spectrum matrix are not used to transform the original time series, as in the previous case. Here, they are applied to an augmented time series involving the original time series and its time derivative. The real part of the new series generated in this manner has properties analogous to those of $z_{i}$ defined by (2).

The time series $u_{j}(t)$ has the spectral representation

$$
u_{j}(t)=\operatorname{Re} \int_{\omega=0}^{\infty} \exp (i \omega t) d A_{j}(\omega)
$$

where $d A_{j}(\omega)$ is a random increment function for an interval $d \omega$ which is uncorrelated with $d A_{j}\left(\omega^{\prime}\right)$ if $\omega^{\prime} \neq \omega$ (e.g. Yaglom, 1962, pp. 37, 81). Formation of the $u_{j}$ correlation matrix with lag $\tau$ gives

$$
\begin{aligned}
\mathrm{U}_{j l}(\tau) & \equiv\left\langle u_{j}(t) u_{l}(t+\tau)\right\rangle \\
& =\frac{1}{2} \operatorname{Re} \int_{\omega=0}^{\infty} \exp (-i \omega \tau)\left\langle d A_{j}(\omega) d A_{l}^{*}(\omega)\right\rangle,
\end{aligned}
$$

where $*$ indicates the complex conjugate. For $\tau=0$, $\mathrm{U}_{j l}(\tau)$ reduces to the covariance matrix defined in (1). The covariance matrix formed by the $d A_{j}(\omega)$ gives the contribution to $\mathbf{U}_{j l}(\tau)$ by the cross spectrum $\boldsymbol{\Phi}_{j l}(\omega)$ in an infinitesimal frequency interval $d \omega$

$$
\boldsymbol{\Phi}_{j l}(\omega) d \omega \equiv \frac{1}{2}\left\langle d A_{j}(\omega) d A_{l}^{*}(\omega)\right\rangle .
$$

We define a filtered time series $u_{j}{ }^{\gamma}(t)$ by removing all spectral components outside the frequency interval $\omega$ to $\omega+d \omega$, i.e.,

$$
u_{j}^{f}(t)=\operatorname{Re}\left[\exp (i \omega t) d A_{j}(\omega)\right] d \omega .
$$

The filtered correlation matrix $\mathrm{U}_{j l} f(t)$ is then

$$
\mathbf{U}_{j l} l^{f}(\tau)=\operatorname{Re}\left[\exp (-i \omega \tau) \boldsymbol{\Phi}_{j l}(\omega)\right] .
$$

Application of the procedures described in the previous section to the filtered time series would involve diagonalizing the symmetric matrix $\mathbf{U}_{j l} f(0)$, or equivalently $\operatorname{Re}\left[\boldsymbol{\Phi}_{j l}(\omega)\right]$. But this approach would discard all the information contained in the quadrature components of the cross spectra.

Since $\boldsymbol{\Phi}_{j l}$ is a Hermitian matrix, we know that it also has a complete set of orthonormal eigenvectors $\mathbf{e}_{i}$ and real eigenvalues $\lambda_{i}$ but now the eigenvectors are complex. In considering how the components of two complex orthogonal modes multiply together to give zero, one must keep in mind not only the mode shapes but also their phase relationships. Let $\mathbf{D}_{i k}(\omega)$ be the diagonal matrix obtained by diagonalizing $\boldsymbol{\Phi}_{j l}(\omega)$ :

$$
\mathbf{D}_{i k}(\omega)=\sum_{j} \sum_{l} e_{i j} e_{k l}{ }^{*} \mathbf{\Phi}_{j l}(\omega) .
$$

If we can find some transformation of our time series that has the diagonal cross spectra given by (13), we have established the desired empirical orthogonal functions. The most obvious choice is the analogue of Eq. (2), which results in a complex transformed series $z_{i}(t)$ since $u_{j}^{f}(t)$ is multiplied by eigenvectors which are complex. Since our main purpose in computing $z_{i}(t)$ is to subject it to further cross-spectrum analysis, it would be more convenient to have a real time series whose power spectrum satisfied (13).

In order to obtain such a series, we first define an augmented (complex) time series from $u_{j}(t)$. Let

$$
w_{j}(t)=\left[u_{j}^{f}(t)-i \omega^{-1} d u_{j}^{f}(t) / d i\right] .
$$

Then the desired transformation is

$$
z_{i}(t)=\operatorname{Re} \sum_{j} e_{i j} w_{j}(t)
$$

To see that (15) has the desired properties, substitute the righthand side of (11) for $u_{j}^{f}(t)$ in (14). For any complex $x, \operatorname{Re}(x)-i \operatorname{Re}(i x)=\operatorname{Re}(x)+i \operatorname{Im}(x)=x$, so that

and

$$
w_{j}(t)=\exp (i \omega t) d A_{j}(\omega),
$$

$$
z_{i}(t)=\operatorname{Re}\left[\exp (i \omega t) \sum_{j} e_{i j} d A_{j}(\omega)\right] .
$$

The unitary transformation determined by the $e_{i j}$ does not change the "length" of but merely "rotates" $d A_{j}(\omega)$ in parameter space. The correlation function for $z_{i}(t)$ using (10) and (13) is simply

$$
\left\langle z_{i}(t) z_{k}(t+\tau)\right\rangle=\delta_{i k} D_{i i}(\omega) \cos (\omega \tau) d \omega
$$

as required.

The empirical orthogonal functions defined by (14) and (15) have properties equivalent to those listed in the previous section. The eigenvectors are orthonormal. The elements of the series (15) have no mutual coherence in the specified frequency band. The $i$ th function has the variance

$$
\left\langle z_{i}{ }^{2}\right\rangle=D_{i i}=\lambda_{i},
$$

and the fraction of the variance of the $j$ th filtered parameter explained by the $i$ th empirical orthogonal 
function is given by the coherence square between $u_{j}$ and $z_{i}$ in the specified frequency band, which can be expressed in terms of the filtered analogue of (7).

\section{Strategy for application and normalization}

The theory given in the previous sections was described in terms of a transformation of a time series where all frequencies had been filtered outside the infinitesimal band $\omega$ to $\omega+d \omega$. The transformation was defined in terms of the eigenvectors of the crossspectrum matrix at $\omega$. A statistically significant crossspectrum matrix determined from a data sample of finite length is necessarily averaged over frequency bands of finite width. If the eigenvector transformation is to be used to study the structure of atmospheric fields over some yet wider range of frequencies, further averaging of the cross-spectrum matrix over this wider frequency range may be desirable. With such averaging, the theory of the previous section is no longer strictly applicable. The time series can be made strictly uncorrelated over a finite frequency range only by applying a different transformation at each different frequency. If, as may be expected, the eigenvectors change little with frequency over the frequency range, a transformation of an averaged cross spectrum should yield a time series which is nearly uncorrelated over the frequency range.

The $u_{j}$ series in the previous sections need not be identified with a single meteorological parameter such as temperature or wind. In certain cases, the different values of the index $j$ may refer to different parameters, each measured in terms of its own characteristic units. In order to insure that the resulting empirical orthogonal function potpourri is not dominated by one particular variable which happens to be expressed in units which give rise to large numerical fluctuations in its time series, some form of normalization is necessary. There are a variety of possible approaches. The simplest of these, and perhaps the least arbitrary, is to divide each $u_{j}$ by the square root of its variance in the specified frequency band. Then all the diagonal elements in the cross-spectrum matrix become equal to unity, and the absolute magnitude of the off-diagonal element $\boldsymbol{\Phi}_{j l}$ becomes equal to the coherence between $u_{j}$ and $u_{l}$ in the specified frequency band.

In the computations for Part II, a number of different normalization schemes were employed. In general, it was found that the results did not depend strongly upon the particular procedure which was followed.

\section{Interpretation}

The linear combination of the $u_{j}^{\prime}$ 's prescribed by a particular mode in an empirical orthogonal function expansion with complex eigenvectors may be thought of as representing a specific wave structure in which the amplitudes and phases of the various $u_{j}^{f}$ are defined by their corresponding eigenvector components $e_{i j}$. The question of whether this structure represents a physical entity, a fabrication of the statistics, or a manifestation of random noise must be decided.

As a basis for this decision it is useful to examine the magnitudes of $\gamma_{i j}$ defined in (7) within any particular mode of the empirical orthogonal function expansion. Since this parameter can be viewed as the coherence square between $u_{j}$ and $z_{i}$ in the frequency band under investigation, we can obtain some indication of its significance by applying the standard test for coherence (e.g., see Amos and Koopmans, 1963). In this way we can single out those $\gamma_{i j}$ 's which are large enough so that the corresponding phase relationships have some meaning. The remaining information may be regarded as noise.

In expansions based on real data, where the number of input series is large, most of the modes have only one or at most a few values of $\gamma_{i j}$ which are statistically significant. Usually these significant phase relationships involve time series of $u_{j}{ }^{f}$ which contain highly redundant information (e.g., wind at adjacent levels). Modes of this type are usually of little physical interest, since they only give reliable phase information on a few of the $u_{j} f^{\prime}$ 's in the expansion.

Having rejected modes of the type described above, we can focus our attention on those modes in which many of the $u_{j}^{f}$ 's are involved in a statistically meaningful way. When such modes exist, they nearly always correspond to the first one or at most to the first few modes in the expansion, since it is these modes which explain the largest fraction of the combined variance of the $u_{j}$ 's in the selected frequency band. By choosing suitably stringent significance criteria, we can assure ourselves that the structure of these few remaining modes is not a result of chance.

Unfortunately, assurance of statistical significance does not necessarily guarantee physical significance. From a statistical point of view, the first few eigenvectors in the expansion are nothing more than those linear combinations of the $u_{j}$ 's which most efficiently represent the structure of the cross spectrum matrix. In order to establish the physical significance of these eigenvectors, it is necessary to show further that the structures which they represent are consistent with governing physical laws. (This applies not only in the case of empirical orthogonal functions, but to the general case in which statistical tools are used in a diagnostic manner to define the structure of physical phenomena.) To this end, it is highly desirable that investigations of this type be conducted in conjunction with synoptic and/or dynamical modeling studies.

Now let us consider a few hypothetical situations in which real wave structures are present in the data. In the simple case where only one wave structure is present in the selected frequency band, superposed on a pattern of random noise, the interpretation of the results is 
straightforward. Normally, in this case, the first mode in the expansion will contain all the relevant information on wave structure, and all the other modes can be rejected on the basis of the criteria mentioned above.

When more than one type of wave structure is present in the selected frequency band, the physical interpretation is somewhat more speculative. It seems to us that a clear separation of the waves into different empirical orthogonal functions may or may not be realized, depending upon the particular choice of $u_{j}$ s used in the expansion. If the wave structures generated by different physical processes in the real atmosphere happen to be mutually orthogonal with respect to the $u_{j}^{f}$ 's [cf. Eq. (5) $]^{3}$ and if they are not coupled with one another [cf. Eq. (3)], then a meaningful separation should be possible. If this condition is not satisfied, then the first mode should represent a compromise structure which incorporates the correlated (non-orthogonal) features of the waves which are present, while the next mode will be that linear combination of $u_{j}^{\prime}$ 's, orthogonal to the first mode, which explains the maximum amount of the residual variance, and so on. In this case, the first mode may still have some physical significance, but it can no longer be identified with one particular wave type. The same problem arises in interpreting results based on cross-spectrum analysis or compositing. When two or more non-orthogonal modes are present in the data the interpretation of the second and higher modes becomes extremely difficult.

In view of these problems that may still exist in the interpretation of the results, one might question whether complex eigenvector analysis really provides any useful information which cannot be readily obtained from simpler analysis techniques. To answer this question, let us consider two situations: one in which the investigator has no a priori knowledge of the wave structures which exist in the data, and the other in which the investigator wishes to test one or more

\footnotetext{
${ }^{3}$ The following simple example is intended to illustrate the meaning of the orthogonality of two wave structures with respect to a specific set of input parameters $u_{j}^{f}$ Let us imagine two superposed zonally propagating waves with identical vertical structure, one propagating westward with a relatively short zonal wavelength, and the other with a much longer wavelength. We will consider data from a limited geographical region which is large enough to encompass at least one full wavelength of the shorter wave, but small enough so that the fluctuations associated with the larger scale wave are essentially simultaneous at all stations in the network. Now if the input parameters $u_{j}^{j}$ consisted of time series of observations made at different levels at the same station, or at two or more stations located at the same longitude, it is clear that the two wave types would be indistinguishable. This is equivalent to saying that the wave structures would have no orthogonality with respect to this particular set of input parameters. If, on the other hand, the input data were time series taken at a pair of stations separated by a distance of $\frac{1}{4}$ wavelength of the shorter wave, then there would be some basis for making a distinction, i.e., some degree of orthogonality. The cross spectra would range from pure real if the long wave amplitude dominates to pure imaginary if the short wave amplitude dominates. In this situation, conventional eigenvector analysis of simultaneous time series would be incapable of detecting the shorter wave.
}

specific hypotheses concerning the structure of the waves.

At a very early stage in an exploratory investigation, it is desirable to establish whether one or more wave structures are present in the frequency band under investigation. The partitioning of the variance in this frequency band among the first few modes in an empirical orthogonal function expansion provides considerable insight into this question. For example, if $\lambda_{1} \gg \lambda_{2}$, this would indicate that one particular wave structure is dominant. If, on the other hand, the first two or more $\lambda$ 's are of comparable size, but significantly larger than the ones which follow, this would indicate more than one type of wave structure may be present. We note that cross-spectrum analysis and compositing do not provide this kind of information. If there are indications that more than one type of wave structure is present in a particular frequency band, then it will probably be necessary to perform a number of expansions based on different input parameters $u_{j}$ in order to obtain a physically meaningful separation. After one has examined the results of several such expansions, he may be in a position to formulate some hypothesis concerning the nature of the observed waves. This brings us to the second situation.

If one has some valid hypothesis concerning the structure of the waves which may be present in a particular frequency band, it should be possible to select a set of $u_{j}$ 's for the expansion which will yield a high degree of orthogonality in the wave structures. An expansion in these parameters can be used to test the hypothesis and to obtain further information on wave structure. In theory, this is rather similar to the approach used by Yanai and Murakami (1970) to separate tropical disturbances into the normal modes on an equatorial beta-plane, and to that used by Wallace (1971) to separate disturbances with differing zonal wavelengths. However, in practice, complex eigenvector analysis involves far fewer assumptions, and it provides a more objective means of testing hypotheses than the methods employed in these other studies. We will show a specific example in Part II.

It seems difficult to conceive of physically distinct waves that do not have some orthogonal degrees of freedom, but the orthogonal variables may not necessarily be present in a given set of observations. From the above discussion, it is apparent that when more than one wave type is present in a particular frequency band, the success of complex eigenvector analysis depends crucially upon the selection of a combination of parameters, $u_{j}$, in which the waves have a substantial degree of orthogonality. If such a combination cannot be found, then the waves will not be distinguishable from one another by this method or, for that matter, by any other method.

The above considerations regarding the interpretation of eigenvector expansions apply to real as well as com- 
plex eigenvector analysis. There is one additional point which arises in connection with the complex expansions. It is important to remember that $z_{i}(l)$ has been formed by transforming the filtered series $u_{j}^{f}$, and thus, it has variance only within the frequency range in which the expansion was performed. In general, a different $z_{i}$ series results from the transformation of the same $u_{j}$ series in each different frequency band.

\section{Summary}

The use of empirical modes determined from the eigenvectors of the cross spectrum matrix has a number of advantages over other techniques previously used in the study of tropical wave disturbances.

1) It provides an objective determination of wave amplitude, even in those parameters which undergo fluctuations which are only partially related to the waves in question.

2) The relative magnitude of the first few eigenvalues in the expansion gives an indication of whether one or more disturbance types are present. The other techniques do not provide a comparable test.

3) It is the most objective means of separating wave disturbances superposed in the same frequency band.

4) The parameter $\gamma_{i j}$, the fraction of the variance of the $j$ th parameter which is explained by the $i$ th mode of the expansion, can be used to assess the statistical significance and relative importance of the various wave structures defined by the expansion.

5) Since this method fully exploits the interrelationships between all the parameters used in the expansion, it should yield results with higher (statistical) significance levels than the other techniques, using the same set of input data. Where statistical significance is marginal, this can be a crucial advantage.
This technique may also have application to many of the types of problems which real eigenvector analysis has conventionally been used. It has the very important advantage of being able to describe wave structures in which certain parameters fluctuate in quadrature with one another. It also makes it possible to discriminate between different types of disturbances on the basis of frequency or wavenumber.

Acknowledgments. We wish to thank Drs. Cecil E. Leith and Paul R. Julian for suggesting a number of substantial improvements in the manuscript. This study was begun while Wallace was on leave of absence to the National Center for Atmospheric Research. The work was also partially supported by the National Science Foundation under Grant GA-629X2.

\section{REFERENCES}

Amos, D. E., and L. H. Koopmans, 1963: Tables of the distribution of the coefficient of coherence for stationary bivariate Gaussian processes. Mono. SCR-483, Sandia Corp., Albuquerque, N. M.

Groves, G. W., and E. J. Hannan, 1968: Time series regression of sea level on weather. Rev. Geophys., 6, 129-174.

Lorenz, E. N., 1956: Empirical orthogonal functions and statistical weather prediction. Sci. Rept. No. 1, Contract AF19(604)1566, Dept. of Meteorology, M. I. T., 49 pp.

Reed, R. J., and E. E. Recker, 1971: Structure and properties of synoptic-scale wave disturbances in the equatorial western Pacific. J. Almos. Sci., 28, 1117-1133.

Wallace, J. M., 1971: Spectral studies of tropospheric wave disturbances in the tropical western Pacific. Rev. Geophys., 9, 557-612.

- 1972: Empirical orthogonal representations of time series in the frequency domain. Part II: Application to the study of tropical wave disturbances. $J$. Appl. Meteor., 11, 893-990.

Yaglom, A. M., 1962: An Introduction to the Theory of Stationary Random Functions. Englewood Cliffs, N. J., Prentice-Hall, $235 \mathrm{pp}$.

Yanai, M., and M. Murakami, 1970: Spectrum analysis of symmetric and antisymmetric equatorial waves. $J$. Meteor. Soc. Japan, 48, 331-347. 